\title{
Broadband Wavelength Conversion Based on On- chip Nonlinear Optical Loop Mirror
}

\author{
Zifei Wang*, ${ }^{1}$ Junjia Wang, ${ }^{1}$ Ivan Glesk, ${ }^{2}$ Lawrence R. Chen ${ }^{1}$ \\ 1. Department of Electrical and Computer Engineering, McGill University, Montreal, Quebec, Canada \\ 2. Electronic and Electrical Engineering, University of Strathclyde Glasgow, Glasgow, Scotland, UK \\ *zifei.wang@mail.mcgill.ca
}

\begin{abstract}
We demonstrate broadband wavelength conversion of $10 \mathrm{~Gb} / \mathrm{s} \mathrm{RZ-OOK}$ signals using a cross phase modulation based integrated nonlinear optical loop mirror on silicon-oninsulator.
\end{abstract}

\section{INTRODUCTION}

Nonlinear optical loop mirror (NOLM) has been studied for several decades and it has many applications in high speed optical signal processing [1], wavelength conversion [2], pulse generation [3], de-multiplexing [4] and so on. On the other side, since silicon is a good nonlinear media, it can be used to generate and process optical signals in low-cost ultracompact chips at high speeds based on nonlinear optical effects [5]. In order to satisfy the need of integration of optical system, in this paper, we firstly designed and fabricated on-chip silicon NOLM and demonstrated the error-free wavelength conversion of $10 \mathrm{~Gb} / \mathrm{s}$ return-to-zero on-off keying (RZ-OOK) signals with a wavelength range from $1535 \mathrm{~nm}$ to $1565 \mathrm{~nm}$ using crossphase modulation (XPM) based NOLM system.

\section{DEVICE AND EXPERIMENTS}

\section{A. XPM based NOLM and Experiments}

The structure we present in this paper is XPM based NOLM, whose principle is explained in [6]. The schematic of integrated NOLM in silicon on insulator (SOI) is shown in Fig 1 (b). The input light is coupled into the integrated silicon chip by vertical grating couplers (VGCs). VGCs are designed for C- band TE mode with a bandwidth of $15 \mathrm{~nm}$ and the coupling loss per VGC is around $-7.5 \mathrm{~dB}$ by measurement. Port 2 and port 3 are connected with a multi-mode interferometer (MMI) coupler with the coupling ratio of 50/50, and the waveguide in the loop is $1.25 \mathrm{~cm}$. The waveguide consists $0.314 \mathrm{~mm}$ bend waveguide with bend radius of $10 \mu \mathrm{m}$ and $12.186 \mathrm{~mm}$ straight waveguide. Port 1 and port 4 are added at the beginning and end of the loop by two $50 / 50 \mathrm{y}$-branch power splitters working as the input port of the pump pulses and port for detection. The continuous-wave $(\mathrm{CW})$ probe is launched through port 2 to the NOLM and port 3 is the output of the device. The device was fabricated using e-beam lithography with a single etch and the size is around $2.65 \mathrm{~mm} \times 0.41 \mathrm{~mm}$. The nonlinear effect occurs in the silicon nanowire loop, whose cross-section is shown in Fig 1 (a). The silicon nanowires have a width of $500 \mathrm{~nm}$ and height of $220 \mathrm{~nm}$, which is designed mostly to support TE fundamental mode. All the components sit on top of buried oxide layer with the thickness of $3 \mu \mathrm{m}$ and are covered by a 2 $\mu \mathrm{m}$ oxide cladding. The measured propagation loss of silicon waveguide is about $-2.7 \mathrm{~dB} / \mathrm{cm}$, so the total loss of the NOLM structure from port 1 to port 3 is measured as around $-27 \mathrm{~dB}$.

The experimental setup is illustrated in Fig 1. The pump is RZ-OOK signals with a PRBS length of $2^{31}-1$, which is generated by a mode lock laser with the frequency of 9.95328 $\mathrm{GHz}$ and the pulse width of $5 \mathrm{ps}$. The pulses are then modulated by a $\mathrm{LiNbO}_{3}$-based Mach-Zehnder modulator (MZ) driven by a pulse pattern generator (PPG). Then the modulated

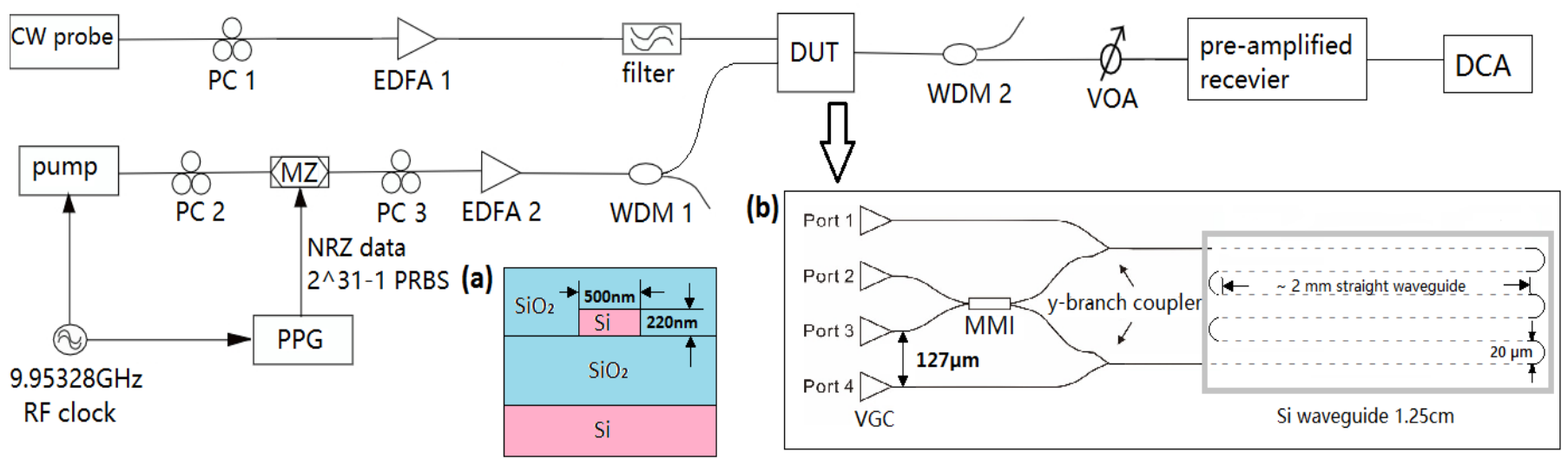

Fig. 1. Schematic of the experimental setup for XPM-based wavelength conversion. Insets: (a) Cross-section of Si nanowire; (b) The structure of integrated NOLM device. PC: polarization controller; EDFA: erbium-doped fiber amplifier; DUT: device under test; VOA: variable optical attenuator. 


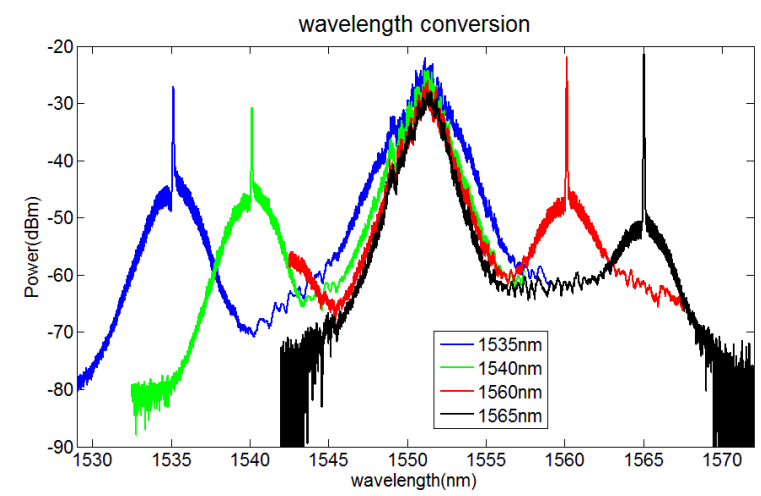

Fig. 2. Spectra at the output of integrated NOLM. The pump is located at $1550 \mathrm{~nm}$, and the probe varies from $1535 \mathrm{~nm}$ to $1565 \mathrm{~nm}$.
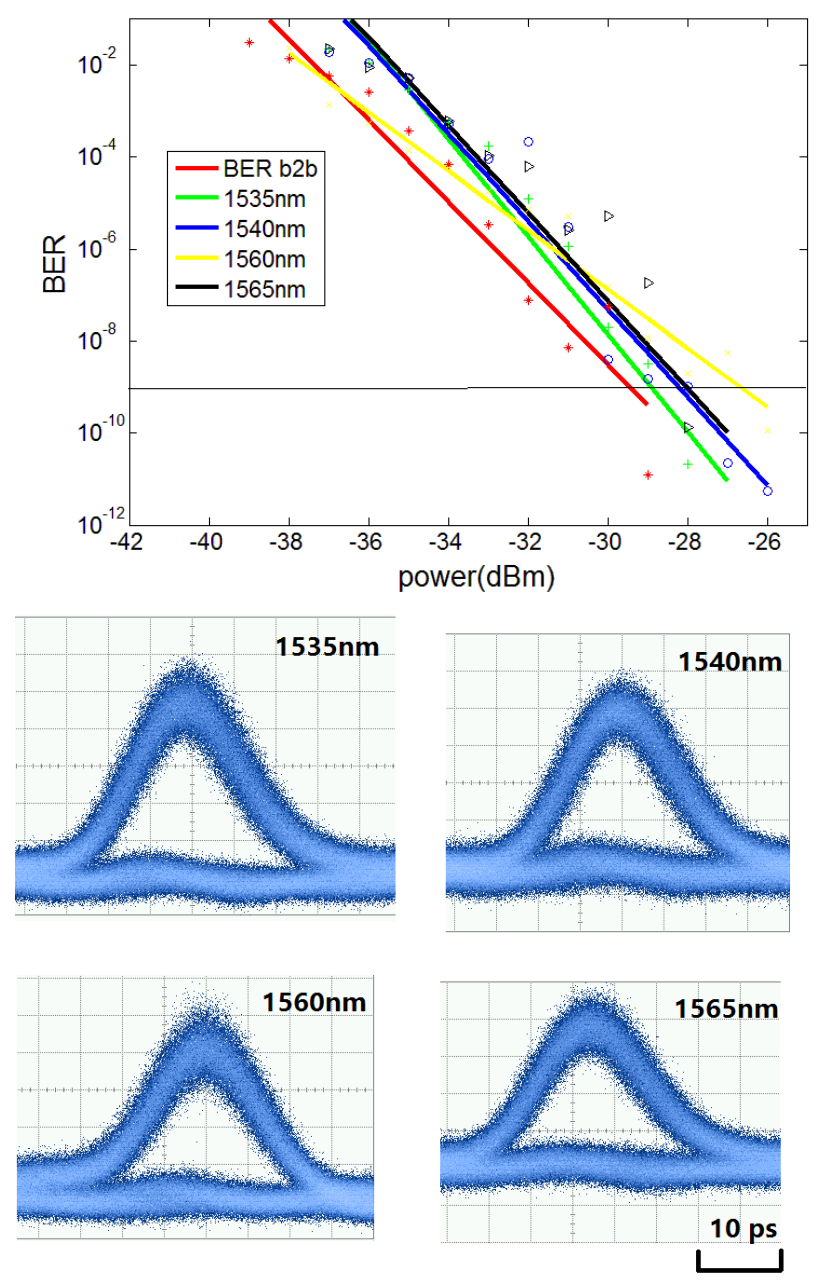

Fig. 3. BER of converted RZ-OOK signals and eye diagrams measured at error-free operation.

pump signals are amplified by a high power EDFA and filtered by a WDM to remove the amplified spontaneous emission (ASE) noise. After amplifying and filtering, the pulses are broadened to $15 \mathrm{ps}$ and therefore the duty cycle of pump signals launched into the chip is $15 \%$. The continuous wave $(\mathrm{CW})$ probe light is also amplified, filtered and coupled into the chip. It combines with the pump signals in the loop to generate XPM effect. The output signals of NOLM are at last filtered by another WDM to remove the pump signals and the wavelength converted signals are received and detected using a preamplified receiver and a digital communications analyzer (DCA). In the experiment, the central wavelength of the pump is fixed to $1550 \mathrm{~nm}$ and we vary the probe wavelength from $1535 \mathrm{~nm}$ to $1565 \mathrm{~nm}$ and bit error rate (BER) measurements are obtained for a probe wavelength of $1535 \mathrm{~nm}, 1540 \mathrm{~nm}$, $1560 \mathrm{~nm}$, and $1565 \mathrm{~nm}$.

\section{B. Results}

Fig. 2 shows the spectral output of the NOLM for different probe wavelengths. The average power into the silicon loop of pump signals and probe are $21 \mathrm{dBm}$ and $18 \mathrm{dBm}$, respectively. We observed XPM-based broadening around the central wavelength of the probe as the probe wavelength is tuned over a $30 \mathrm{~nm}$ range.

In order to verify the performance of wavelength conversion, we measure the BER of the wavelength converted signals, see Fig. 3. We obtained error free operation and observed a clearly opened eye-diagram in a wavelength range of $30 \mathrm{~nm}$ with the power penalties of less than $2.8 \mathrm{~dB}$.

\section{CONCLUSION}

We have presented the integrated NOLM on SOI platform for the first time and successfully demonstrated XPM-based wavelength conversion for $10 \mathrm{~Gb} / \mathrm{s}$ RZ-OOK signals. The converted RZ-OOK signal exhibits no error floor with a power penalty less than $2.8 \mathrm{~dB}$. We believe that XPM-based wavelength conversion in an integrated NOLM will provide an advancement toward the integration of all-optical high-speed optical networking systems.

\section{ACKNOWLEDGMENT}

This project has received funding from the European Union's Horizon 2020 research and innovation programme under the Marie Skłodowska-Curie grant agreement No 734331 .

\section{REFERENCES}

[1] A. Bogoni, "Nonlinear optical loop mirrors: investigation solution and experimental validation for undesirable counterpropagating effects in all-optical signal processing, " IEEE J. Sel. Topics in Quantum Electron. 10,5 (2004): 1115-1123.

[2] T. Sakamoto et al, "All-optical wavelength conversion of 500-fs pulse trains by using a nonlinear-optical loop mirror composed of a highly nonlinear DSF, ” IEEE Photon. Technol. Lett. 13,5 (2001): 502-504.

[3] T. Huanget al, "Photonic generation of UWB pulses using a nonlinear optical loop mirror and its distribution over a fiber link, " IEEE Photon. Technol. Lett. 23,17 (2011): 1255-1257.

[4] B. G. Lee et al, "All-optical regeneration and demultiplexing for 160 $\mathrm{Gb} / \mathrm{s}$ transmission systems using a NOLM-based three-stage scheme," IEEE J. Sel. Topics in Quantum Electron. 10,1 (2004): 192-196.

[5] J. Leuthold et al., "Nonlinear silicon photonics," Nat. Photonics 4" (2010): 535.

[6] A. Bononi et al., "Nonlinear optical loop mirrors: inverstigation solution an experimental validation for undesirable counterpropagating effects in all-optical signal processing," IEEE J. Sel. Topics in Quantum Electron. 10,5 (2004): 1115-1123. 
